\title{
OR-9
}

\section{Antihyperlipidemic Activity of Methanolic and Aqueous Extracts of Garcinia Mangostana in Poloxamer 407-Induced Acute Hyperlipidemic Rats}

\author{
Manimegalai Manogaran, Mohd Zaini Asmawi and Vikneswaran Murugaiyah* \\ Discipline of Pharmacology, School of Pharmaceutical Sciences, Universiti Sains Malaysia, 11800 Penang, Malaysia; \\ E-mail:vicky@usm.my
}

Garcinia mangostana is a tropical plant found commonly in South East Asia. This plant possesses anti-inflammatory, antibacterial, antiulcer, antiseptic and also showed prevention against oxidative damage of low density lipoprotein (LDL). The present study aimed to evaluate the antihyperlipidemic activity of the methanolic and aqueous extracts of various parts of G. mangostana in poloxamer 407- induced hyperlipidemic rats. The aqueous extracts exhibited larger reduction in total cholesterol, triglycerides, and LDL and high density lipoprotein (HDL) levels compared to methanolic extracts. Aqueous extract of leaves showed significant reduction of $62.95 \%$ and $82.08 \%$ in total cholesterol $(p<0.001)$ and triglycerides $(p<0.001)$ levels, respectively compared to hyperlipidemic control at 58 hour after hyperlipidemia induction. Likewise, aqueous extract of stem exhibited significant reduction of $74.97 \%$ and $78.50 \%$ in total cholesterol $(\mathrm{p}<0.001)$ and triglycerides $(\mathrm{p}<0.001)$ levels, respectively at 58 hour after hyperlipidemia induction. In conclusion, aqueous extract of stem showed highest reduction of total cholesterol while aqueous extract of leaves showed highest reduction of triglycerides compared to hyperlipidemic control group. These results warrant further investigation on lipids lowering capacity of these extracts in the high fat diet-induced chronic hyperlipidemic rat model.

Keywords: Antihyperlipidemic, Poloxamer 407, Garcinia mangostana, total cholesterol, triglycerides. 